\title{
On the Growth of Meromorphic Solutions of Linear Differential Equations Having Arbitrary Entire Coefficients $(*)$.
}

\author{
STEVEn B. BANK (Urbana, Illinois, U.S.A)
}

\begin{abstract}
Summary. - We investigate the class of linear ordinary differential equations having arbitrary entire functions as coefficients. In our main results, we obtain estimates for the growth of meromorphic solutions of such equations.
\end{abstract}

\section{1. - Introduction.}

In this paper, we treat $n$-th order homogeneous linear differential equations,

$$
L(y)=\sum_{j=0}^{n} f_{j}(z) y^{(j)}=0
$$

where the coefficients $f_{0}, f_{1}, \ldots, f_{n}$ are arbitrary entire functions.

In [11], G. ValInoN used the Valiron-Wiman theory [12; Chapter IV] to prove that when all the coefficients $f_{i}$ are polynomials, then any entire solution of $L(y)=0$ is of finite order of growth. It easily follows that when all the coefficients $f_{i}$ are polynomials (and thus $f_{n}$ has only finitely many zeros), then every solution of $L(y)=0$ which is meromorphic in the plane must also be of finite order of growth.

In the case when one or more of the coefficients $f_{\hat{\sigma}}$ are transcendental entire functions, the Valiron-Wiman theory can still be used to estimate the growth of an entire solution in terms of the growth of the coefficients. (In [6; pp. 202-205], M. FRer obtained this estimate in the case when $f_{n}$ has no zeros. In the case when $f_{n}$ has zeros, an easy modification of the proof, using a minimum modulus estimate on $f_{n}$ which holds "nearly everywhere " (see $\S 2$ below) such as in $[10 ;$ p. 336] if $f_{n}$ is of finite order or $\left[4 ;\right.$ p. 97] if $f_{n}$ is of infinite order, also leads to an estimate on the growth of entire solutions in terms of the growth of the coefficients.)

In the case when one or more of the coefficients $f_{i}$ are transcendental, the problem of determining the growth of meromorphic solutions in the plane of $L(y)=0$ is more complicated than for entire solutions since the Valiron-Wiman theory is not applicable. The difference is indicated by an example constructed in [3] which shows

(*) Entrata in Redazione il 20 dicembre 1974. 
that even in the first-order case, it is not possible to estimate the growth of a meromorphic solution in terms of the growth of the coefficients alone. (It was shown in [3] that given any increasing function $\Phi(r)$ on $(0,+\infty)$, there exists a meromorphic function $h(z)$ in the plane, which is a solution of a first-order linear differential equation $f_{1} y^{\prime}+f_{0} y=0$ whose coefficients $f_{0}$ and $f_{1}$ are entire functions of finite order, such that the Nevanlinna characteristic $T(r, h)$ of $h(z)$ is larger than $\Phi(r)$ at a sequence of $r$ tending to $+\infty$. We remark that although a pole of the solution $h$ must be a zero of the coefficient $f_{1}$, the multiplicity of the pole of $h$ may far exceed the multiplicity of the zero of $f_{1}$ ). It was shown in [1] that given any firstorder differential equation $\Omega\left(z, y, y^{\prime}\right)=0$, where $\Omega$ is a polynomial in $y$ and $y^{\prime}$ whose coefficients are arbitrary entire functions of $z$, then the growth of a meromorphic solution of $\Omega=0$ can be estimated in terms of the growth of the coefficients of $\Omega$ and the counting function for the poles of the solution. (The fact that the counting function for the poles cannot be eliminated from the estimate for the growth is indicated by the example of unrestricted growth described above.)

In the case of equations of order higher than one, it was shown in [2] that if $\Omega\left(\approx, y, y^{\prime}, \ldots, y^{(n)}\right)$ is a polynomial in $y, y^{\prime}, \ldots, y^{(n)}$ whose coefficients are arbitrary entire functions of $z$, then the growth of any meromorphic solution $h(z)$ of $\Omega=0$ can be estimated in terms of the growth of the coefficients of $Q$ and (i) the counting functions for the zeros and poles of $h$ if $n=2$, or (ii) the counting functions for the zeros and poles of $h$ and the counting functions for the zeros of certain successive logarithmic derivatives, $h_{1}=h^{\prime} / h, \ldots, h_{k}=h_{k-1}^{\prime} / h_{k-1}$, for some $k \leqslant n-2$, if $n>2$. In the present paper, we show that in the special case of linear differential equations, these results can be greatly improved. In $\S 3$ below, we show that for a secondorder homogeneous linear differential equation having arbitrary entire coefficients, the growth of a meromorphic solution can be estimated in terms of the growth of the coefficients and the counting function for the zeros of the solution. (Of course, the example of unrestricted growth described earlier indicates that the counting function for the zeros cannot be eliminated from the estimate.) For homogeneous linear differential equations of order higher than two, we show in $\$ 5$ that regardless of the order of the equation, the growth of a meromorphic solution $h(z)$ can be estimated in terms of the growth of the coefficients and the counting functions for the zeros of $h$ and for the distinct zeros of $h^{\prime} / h$. In $\$ 7$, we show that for a homogeneous linear differential equation of any order $n$, the growth of a meromorphic solution $h$ can be estimated in terms of the growth of the coefficients and the counting function for the distinct zeros of $h(z)-z^{m}$, for some $m$ in the set $\{0,1, \ldots, n\}$. This result is a corollary of a theorem proved in $\S 6$, which states that the growth of a meromorphic solution of the nonhomogeneous $n$-th order linear differential equation $L(y)=f$ (where $f$ is entire, $f \neq 0$ ), can be estimated in terms of the growth of the coefficients $f, f_{0}, \ldots, f_{n}$ and the counting function for the distinct zeros of the solution.

In $\S 8$, we investigate the growth of meromorphic solutions of a special class of $n$-th order linear differential equations which can be reduced to second-order equations. 


\section{2. - Notation.}

For a meromorphic function $g(z)$ on the plane, we will use the standard notation for the Nevanlinna functions $m(r, g), N(r, g)$ and $T(r, g)$ introduced in $[9 ; \mathrm{pp} .6,12]$. We will also use the notation $n(r, g)$ to denote the number of poles (counting multiplicity) of $g$ in $|z| \leqslant r$, and we will denote by $\bar{n}(r, g)$ the number of distinct poles of $g$ in $|z| \leqslant r$. As in $[9 ; p .70]$, we will denote by $\bar{N}(r, g)$, the function obtained by replacing $n(r, g)$ by $\bar{n}(r, g)$ in the definition of $N(r, g)$. As in [2], we will say that a certain property $P(r)$ holds "nearly everywhere in $[0,+\infty)$ " if $P(r)$ holds for all $r \geqslant 0$ with the possible exception of a set of finite measure.

3. - THeorem 1. - Let $f_{0}(z), f_{1}(z)$ and $f_{2}(z)$ be entire functions. Let $M_{1}(r)$ be a monotone nondecreasing function on $[0,+\infty)$ and let $A(r)$ be a monotone nonincreasing function on $[0,+\infty)$ such that $M_{1}(r) \geqslant 1$ and $A(r)>0$ for all $r \geqslant 0$. Let

$$
\left|f_{j}(z)\right| \leqslant M_{1}(r) \quad \text { on }|z|=r \text { for } j=0,1,2 \text { and all } r>0 \text {. }
$$

In addition, let

$$
\left|f_{2}(z)\right| \geqslant A(r) \quad \text { on }|z|=r \text {, nearly everywhere in }[0,+\infty) .
$$

Let $y(z)$ be a meromorphic function on the plane which is not identically zero and which satisfies the equation,

$$
f_{3}(z) y^{\prime \prime}(z)+f_{1}(z) y^{\prime}(z)+f_{0}(z) y(z)=0
$$

at each point where $y$ is analytic. Then for any $a>1$, there exist positive constants $K, K_{1}$ and $r_{0}$ such that for all $r>r_{0}$,

$$
T(r, y) \leqslant K\left(r N(a r, 1 / y)+r^{2} \exp \left(K_{1} G(a r) \log ^{+} G(a r)\right)\right),
$$

where,

$$
G(r)=r \bar{N}(r, 1 / y)+r^{2} M_{1}(r) / A(r)
$$

Thus, the growth of the solution $y$ can be estimated in terms of the growth of the coefficients in the equation and the counting function $N(r, 1 / y)$ for the zeros of $y$.

Proor. - Given $a>1$, let $\sigma>1$ be such that $\sigma^{2}=a$. Let $w=y^{\prime} / y$. Then $w$ satisfies the first-order equation,

$$
f_{2} w^{2}+f_{2} w^{\prime}+f_{1} w+f_{0}=0
$$


In view of $(2)$ and $(3)$, it follows from $[1 ; \S 3]$ or $[2 ; \S 3]$ that there exist positive constants $K_{2}$ and $r_{1}>1$ such that for all $r>r_{1}$,

$$
T(r, w) \leqslant K_{2}(J(\sigma r))
$$

where,

$$
J(r)=\log ^{+} M_{1}(r)+r^{2} M_{1}(r) / A(r)+r N(r, w) .
$$

Olearly $n(t, w)=\bar{n}(t, y)+\bar{n}(t, 1 / y)$ for all $t \geqslant 0$, so it easily follows that,

$$
N(r, w)=\bar{N}(r, y)+\bar{N}(r, 1 / y) \quad \text { for all } r>0 .
$$

Since the coefficients $f_{0}, f_{1}$ and $f_{2}$ are entire, it follows easily from (4) that if $y$ has a pole at a point $z_{0}$, then $f_{2}$ must have a zero at $z_{0}$. Of course, the multiplicity of the pole of $y$ may far exceed the multiplicity of the zero of $f_{2}$, but in any case we have $\bar{n}(t, y) \leqslant \bar{n}\left(t, 1 / f_{2}\right)$ for all $t \geqslant 0$. It easily follows that there is a constant $K_{3} \geqslant 0$ such that

$$
\vec{N}(r, y) \leqslant \vec{N}\left(r, 1 / f_{2}\right)+K_{3} \quad \text { for all } r>0 .
$$

Now by Jensen's formula, it follows $[9 ; p .11]$ that $T\left(r, 1 / f_{2}\right)=T\left(r, f_{2}\right)+O(1)$ as $r \rightarrow \infty$, and since $f_{2}$ is entire, it thus follows from (2), $[9 ; \mathrm{p} .24]$ and $(11)$, that there are positive constants $r_{2}>r_{1}$ and $K_{4}$ such that

$$
\bar{N}(r, y) \leqslant \log ^{+} M_{1}(r)+K_{4} \quad \text { for all } r>r_{2} .
$$

Let $r_{3}>1$ be a value of $r$ at which (2) and (3) are valid. Then clearly $M_{1}\left(r_{3}\right) / A\left(r_{3}\right) \geqslant 1$, and since $M_{1} / A$ is nondecreasing we have $M_{1}(r) / A(r) \geqslant 1$ for all $r \geqslant r_{3}$. Thus there exists $r_{4}>r_{2}$ such that,

$$
r K_{1} \leqslant r^{2} M_{1}(r) / A(r) \quad \text { for } r>r_{4} .
$$

Now $\log ^{\dagger} M_{1}(r) \leqslant M_{1}(r)$ for all $r \geqslant 0$, and since $A(r)$ is nonincreasing, we thus have,

$$
\log ^{+} M_{1}(r) \leqslant A\left(r_{4}\right) M_{1}(r) / A(r) \quad \text { for } r \geqslant r_{4}
$$

Letting $r_{5}>r_{4}$ be a value of $r$ for which $(r+1) A\left(r_{4}\right)+2 r^{2} \leqslant 3 r^{2}$ if $r>r_{5}$, it now follows from (9), (10), (12), (13) and (14) that for all $r>r_{5}$,

$$
J(r) \leqslant 3 G(r),
$$

where $G(r)$ is as in $(6)$. 
Now let $u=1 / y$ and let $v=-w$. Then $v=u^{\prime} / u$, so by [2; $\left.\$ 7\right]$, there exist positive constants $r_{6}>r_{5}, C$ and $C_{1}$ such that for all $r>r_{6}$,

$$
T(r, u) \leqslant C(r N(\sigma r, u))+r^{2} \exp \left(C_{1} \Psi(\sigma r)\right)
$$

where,

$$
\Psi(r)=T(r, v)+N(r, v)\left(\log r+\log ^{+} N(r, v)\right)
$$

Since $v=-w$, clearly there exists $r_{7}>r_{6}$ such that,

$$
\Psi(r) \leqslant T(r, w)\left(2 \log r+\log ^{+} T(r, w)\right) \quad \text { for } r>r_{7}
$$

In view of (8) and (15) and the fact that $G(r) \geqslant r^{2}$ for $r>r_{3}$ (since $M_{1}(r) / A(r) \geqslant 1$ for $\left.r>r_{3}\right)$, it follows from (18), that there exists $r_{8}>r_{7}$ such that,

$$
\Psi(r) \leqslant 12 K_{2} G(\sigma r) \log ^{+} G(\sigma r) \quad \text { for all } r>r_{8}
$$

Since $T(r, y)=T(r, u)+O(1)$ by Jensen's formula and since $\sigma^{2}=a$ and $N(\sigma r, u) \leqslant$ $\leqslant N(a r, u)$, it easily follows from (16) and (19) that there exists $r_{0}>r_{8}$ such that (5) holds for all $r>r_{0}$, where $K=2 C$ and $K_{1}=12 K_{2} C_{1}$. This completes the proof of Theorem 1.

4. - Lemma. - Let $\Omega\left(z, y, y^{\prime}, \ldots, y^{(n)}\right)$ be a polynomial in $y, y^{\prime}, \ldots, y^{(n)}$, whose coefficients are meromorphic functions of $z$ in the plane. For each $r>0$, let $\Delta(r)$ be the maximum of the Nevanlinna characteristics of the coefficients of $\Omega$. For each nonnegative integer $q$, let $\Omega_{q}$ denote the homogeneous part of $\Omega$ of total degree $q$ in the indeterminates $y, y^{\prime}, \ldots, y^{(n)}$. Let $y_{0}(z)$ be a meromorphic function in the plane which is not identically zero and which satisfies the equation $\Omega=0$, but which for some nonnegative integer $q$ does not satisfy the equation $\Omega_{q}=0$. Then for any $a>1$, there exist positive constants $K$ and $r_{0}$ such that for all $r>r_{0}$, we have

$$
T\left(r, y_{0}\right) \leqslant K(F(a r))
$$

where,

$$
F(r)=\bar{N}\left(r, 1 / y_{0}\right)+\bar{N}\left(r, y_{0}\right)+\Delta(r)+\log r
$$

Proof. - Let $w_{0}=y_{0}^{\prime} / y_{0}$. Then it is easy to see by induction (see [7; p. 73]), that for each $j \geqslant 1, y_{0}^{(j)} / y_{0}$ can be written as a polynomial in $w_{0}, w_{0}^{\prime}, \ldots, w_{0}^{(j-1)}$ with nonnegative integer coefficients. It follows that for each nonnegative integer $q, \Omega_{q}\left(z, y_{0}(z), \ldots, y_{0}^{(z)}(z)\right) /\left(y_{0}(z)\right)^{\alpha}$ can be written $R_{q}\left(z, w_{0}(z), \ldots, w_{0}^{(z-1)}(z)\right)$, where $R_{q}\left(z, w_{1}, \ldots, w_{n}\right)$ is a polynomial in $w_{1}, \ldots, w_{n}$, whose coefficients are linear combina- 
tions (with constant coefficients) of the coefficients of $\Omega_{q}$. If $m$ is the maximum nonnegative integer with the property that $y_{0}$ is not a solution of the equation $\Omega_{m}=0$, then since $y_{0}$ satisfies $\Omega=0$, we have

$$
\sum_{\alpha=0}^{m} R_{\alpha}\left(z, w_{0}(z), \ldots, w_{0}^{(n-1)}(z)\right)\left(y_{0}(z)\right)^{\alpha} \equiv 0
$$

at each point where $y_{0}$ is not zero or infinity. Using the elementary rules for calculating with the Nevanlinna characteristic $[9 ;$ p. 14], it follows $[8 ;$ p. 108] that,

$$
T\left(r, y_{0}\right) \leqslant \sum_{q=0}^{m} T\left(r, R_{q}\left(z, w_{0}, \ldots, w_{0}^{(n-1)}\right)\right)+O(1)
$$

as $r \rightarrow+\infty$. Now by $[7 ;$ p. 55], for each $j \geqslant 1$,

$$
T\left(r, w_{0}^{(j)}\right)=O\left(T\left(r, w_{0}\right)\right),
$$

nearly everywhere in $[0,+\infty)$ as $r \rightarrow+\infty$. In addition, by $[9 ; p .63]$ and $(10)$, we have,

$$
T\left(r, w_{0}\right)=O\left(\bar{N}\left(r, y_{0}\right)+\bar{N}\left(r, 1 / y_{0}\right)+\log \left(r T\left(r, y_{0}\right)\right)\right)
$$

nearly everywhere in $[0,+\infty)$ as $r \rightarrow+\infty$. Using the elementary rules for calculating with the Nevanlinna characteristic $[9 ;$ p. 14], and recalling the definitions of $R_{q}$ and $\Delta(r)$, it follows easily from (23), using (24) and (25), that there is a constant $K$ such that $(20)$ holds with $a=1$, nearly everywhere in $[0,+\infty)$. Since $T\left(r, y_{0}\right)$ and $F(r)$ are monotone nondecreasing functions, the conclusion of the lemma follows immediately from either $[1$; Lemma $O$, p. 68] or $[2 ; \S 2]$.

5. - Theorem 2. - Let $f_{0}(z), f_{1}(z), \ldots, f_{n}(z)$ be entire functions and let $f_{n}$ be not identically zero. For each $r>0$, let $\Phi(r)$ denote the maximum of $\left\{T\left(r, f_{j}\right)\right.$ : $j=0, \ldots, n\}$. Let $y_{0}(z)$ be a nonconstant meromorphic function on the plane which satisfies the linear differential equation,

$$
\sum_{j=0}^{n} f_{j} y^{(j)}=0
$$

at each point where $y_{0}$ is analytic. Let $w_{0}=y_{0}^{\prime} / y_{0}$. Then for any $a>1$, there exist positive constants $K, K_{1}$ and $r_{0}$ such that for all $r>r_{0}$,

$$
T\left(r, y_{0}\right) \leqslant K\left(r N\left(a r, 1 / y_{0}\right)+r^{2} \exp \left[K_{1} H(a r) \log (r H(a r))\right]\right)
$$


where,

$$
H(r)=\bar{N}\left(r, 1 / y_{0}\right)+\bar{N}\left(r, 1 / w_{0}\right)+\Phi(r)+\log r
$$

Thus the growth of the solution $y_{0}$ can be estimated in terms of the growth of the coefficients and the counting functions for the zeros of $y_{0}$ and the distinct zeros of $y_{0}^{\prime} / y_{0}$.

Proof. - Since $y_{0}$ satisfies equation (26), it is clear that $w_{0}$ satisfies an equation,

$$
\Lambda\left(z, w, w^{\prime}, \ldots, w^{(n-1)}\right)=0
$$

where $\Lambda$ is a polynomial in $w, w^{\prime}, \ldots, w^{(n-1)}$, whose homogeneous part of maximum total degree in $w, w^{\prime}, \ldots, w^{(n-1)}$ is $f_{n} w^{n}$, and where the coefficients of $\Lambda$ are linear combinations (with integer coefficients) of $f_{0}, \ldots, f_{n}$. Thus, if $a>1$, and we let $a>1$ be such that $\sigma^{2}=a$, then by $\S 4$, there exist positive constants $K_{2}$ and $r_{1}$, such that for all $r>r_{1}$, we have

$$
T\left(r, w_{0}\right) \leqslant K_{2} F(\sigma r)
$$

where

$$
F^{\prime}(r)=\bar{N}\left(r, 1 / w_{0}\right)+\bar{N}\left(r, w_{0}\right)+\Phi(r)+\log r
$$

Since the coefficients of (26) are entire, it is easy to see that if $y_{0}$ has a pole at a point $z_{0}$, then $f_{n}$ must have a zero at $z_{0}$. Thus $\bar{n}\left(t, y_{0}\right) \leqslant \bar{n}\left(t, 1 / f_{n}\right)$ for all $t \geqslant 0$. From this and Jensen's formula (i.e. $T\left(r, 1 / f_{n}\right)=T\left(r, f_{n}\right)+O(1)$ ), it follows using the definition of $\Phi(r)$, that there are positive constants $r_{2}>r_{1}$ and $K_{3}$ such that for $r>r_{2}$,

$$
\bar{N}\left(\sigma r, y_{0}\right) \leqslant \Phi(\sigma r)+K_{3} .
$$

Since $\bar{N}\left(r, w_{0}\right)=\bar{N}\left(r, y_{0}\right)+\bar{N}\left(r, 1 / y_{0}\right)$ (see (10)), it easily follows from (30), (31) and (32), that there exists $r_{3}>r_{2}$ such that for all $r>r_{3}$,

$$
T\left(r, w_{0}\right) \leqslant 2 K_{2} H(\sigma r),
$$

where $H$ is given by (28).

Now let $u=1 / y_{0}$ and $v=-w_{0}$. Then $v=u^{\prime} / u$, so by $[2 ; \S 7]$, there exist positive constants $r_{6}>r_{3}, C$ and $C_{1}$ such that (16) holds for $r>r_{6}$, where $\Psi(r)$ is given by (17) and thus obeys (18) (where $w$ is the function $w_{0}$ ) for all $r$ greater than some number $r_{7}>r_{6}$. Since $\sigma^{2}=a$, it easily follows from (18) and (33) that there exists $r_{8}>r_{7}$ such that for all $r>r_{8}$, we have,

$$
\Psi(\sigma r) \leqslant 6 K_{2} H(a r) \log (r H(a r))
$$


Since $T\left(r, y_{0}\right)=T(r, u)+O(1)$, it easily follows from (16) and (34) that (27) holds for all $r$ greater than some number $r_{0}$, where $K=2 C$ and $K_{1}=6 K_{2} C_{1}$. This concludes the proof of Theorem 2.

REMARK. - With the notation as in Theorem 2 , it is clear that $\bar{n}\left(t, 1 / w_{0}\right) \leqslant \bar{n}\left(t, 1 / y_{0}^{\prime}\right)$ for all $t \geqslant 0$. Tt follows that the estimate (27) is valid for all sufficiently large $r$ if $H(r)$ is replaced by,

$$
H_{1}(r)=\bar{N}\left(r, 1 / y_{0}\right)+\vec{N}\left(r, 1 / y_{0}^{\prime}\right)+\Phi(r)+\log r .
$$

6. - THEOREM 3. - Let $f, f_{0}, \ldots, f_{n}$ be entire functions and let $f$ and $f_{n}$ be not identically zero. For each $r>0$, let $\Delta(r)$ be the maximum of the numbers $T(r, f)$, $T\left(r, f_{0}\right), \ldots, T\left(r, f_{n}\right)$. Let $y_{0}(z)$ be a meromorphic function on the plane which is not identically zero and which satisfies the nonhomogeneous linear differential equation,

$$
\sum_{j=0}^{n} f_{i} y^{(j)}=f
$$

at each point where $y_{0}$ is analytic. Then for any $a>1$, there exist positive constants $r_{0}$ and $K_{0}$ such that for all $r>r_{0}$, we have

$$
T\left(r, y_{0}\right) \leqslant K_{0}\left(\bar{N}\left(a r, 1 / y_{0}\right)+\Delta(a r)+\log r\right) .
$$

Thus the growth of the solution $y_{0}$ of the nonhomogeneous equation can be estimated in terms of the growth of the coefficients and the counting function for the distinct zeros of $y_{0}$.

Proof. - By $\S 4$, if $a>1$, there are constants $K$ and $r_{1}$ such that for all $r>r_{1}$, inequality (20) holds where $F(r)$ is given by (21). Since the coefficients of (36) are entire functions, clearly if $y_{0}$ has a pole at a point $z_{0}$, then $f_{n}$ has a zero at $z_{0}$. Thus, $\bar{n}\left(t, y_{0}\right) \leqslant \bar{n}\left(t, 1 / f_{n}\right)$ for all $t \geqslant 0$.

From this and Jensen's formula, it follows (as in (32)) using the definition of $\Delta(r)$ that for all $r$ greater than some number $r_{2}$, we have

$$
\bar{N}\left(a r, y_{0}\right) \leqslant \Delta(a r)+K_{1},
$$

where $K_{1}$ is a constant. In view of $(20),(21)$ and (38), it is clear that (37) holds for all $r$ greater than some number $r_{0}$, if we take $K_{0}=2 K$. This concludes the proof of Theorem 3.

7. - Corollary. - Let $f_{0}, f_{1}, \ldots, f_{n}$ be entire functions and let $f_{n}$ be not identically zero. For each $r>0$, let $\Phi(r)$ denote the maximum of the numbers $T\left(r, f_{0}\right), \ldots$, 
$T\left(r, f_{n}\right)$. Let $y_{0}(z)$ be a meromorphie function on the plane which is not identically zero and which satisfies the linear differential equation,

$$
L(y)=\sum_{j=0}^{n} f_{j} y^{(j)}=0
$$

at each point where $y_{0}$ is analytic. Then,

(A) If $g$ is any entire function which is not a solution of equation (39), then for any $a>1$, there exist positive constants $K$ and $r_{0}$, such that for all $r>r_{0}$, we have

$$
T\left(r, y_{0}\right) \leqslant K W(a r),
$$

where

$$
W(r)=\bar{N}\left(r, 1 /\left(y_{0}-g\right)\right)+\Phi(r)+T(r, g)+\log r .
$$

$(B)$ There exists a nonnegative integer $m \leqslant n$ with the property that for any $a>1$, there exist positive constants $K$ and $r_{0}$ such that for all $r>r_{0}$ we have,

$$
T\left(r, y_{0}\right) \leqslant K\left(\bar{N}\left(a r, 1 /\left(y_{0}-z^{m}\right)\right)+\Phi(a r)+\log r\right) .
$$

Proof. - Part (A). Let $g$ be an entire function such that $L(g) \neq \equiv$. Let $u_{0}=y_{0}-g$. Then $u_{0}$ solves the nonhomogeneous equation,

$$
L(u)=-L(g) .
$$

In view of the definition of $\Phi(r)$ and the fact that for $j \geqslant 1, T\left(r, g^{(j)}\right)=O(T(r, g))$ nearly everywhere in $[0,+\infty)$ as $r \rightarrow \infty$ by $[7 ;$ p. 55], it easily follows that there is a constant $K_{1}>1$ such that nearly everywhere in $[0,+\infty)$,

$$
T(r,-L(g)) \leqslant K_{1}(\Phi(r)+T(r, g)+1) .
$$

Let $a>1$, and let $\sigma>1$ be such that $\sigma^{2}=a$. Since both sides of (44) are monotone nondecreasing on $(0,+\infty)$, it follows from $\left[1 ;\right.$ p. 68], that there exists $r_{1}>0$ such that for all $r>r_{1}$, we have

$$
T(r,-L(g)) \leqslant K_{1}(\Phi(\sigma r)+T(\sigma r, g)+1) .
$$

Hence if we denote by $\Delta(r)$ the maximum of the characteristics of coefficients of the equation (43), then since $K_{1}>1$, it follows from (45) and the definition of $\Phi(r)$ that,

$$
\Delta(r) \leqslant K_{1}(\Phi(\sigma r)+T(\sigma r, g)+1) \text { for } r>r_{1} .
$$


But by Theorem $3(\S 6)$, there exist positive constants $r_{2}>r_{1}$ and $K_{2}$ such that for $r>r_{2}$,

$$
T\left(r, u_{0}\right) \leqslant K_{2}\left(\bar{N}\left(\sigma r, 1 / u_{0}\right)+\Delta(\sigma r)+\log r\right) .
$$

Since $T\left(r, y_{0}\right) \leqslant T\left(r, u_{0}\right)+T(r, g)+O(1)$, the conclusion of Part $(A)$ now follows immediately from (47) and (46).

Part $(B)$. It is impossible that all of the $n+1$ functions $1, z, z^{2}, \ldots, z^{n}$ be solutions of equation (39), since the $n$-th order equation (39) cannot have more than $n$ linearly independent solutions in a neighborhood of a point where $f_{n}$ does not vanish. Thus (40) holds with $g=z^{m}$ for some $m$ in $\{0,1, \ldots, n\}$. Since $T\left(r, z^{m}\right)=m(\log r)$ for $r>1$, Part $(B)$ clearly follows from (40).

8. - THEOREM 4. - Let $f_{0}, f_{1}$ and $f_{2}$ be entire functions. Let $M_{1}(r)$ and $A(r)$ be as in the hypothesis of Theorem 1 (\$3) satisfying (2) and (3). Let $y_{0}(z)$ be a transcendental meromorphic function on the plane which satisfies the equation,

$$
f_{2} y_{0}^{(n)}+f_{1} y_{0}^{(n-1)}+f_{0} y_{0}^{(n-2)}=0,
$$

at each point where $y_{0}$ is analytic. Then for any $a>1$, there exist positive constants $C, C_{\mathrm{x}}$ and $r_{0}$ such that for all $r>r_{0}$, we have

$$
T\left(r, y_{0}\right) \leqslant O\left(r N\left(a r, 1 / y_{0}^{(n-2)}\right)+r^{2} \exp \left(C_{1} J(a r) \log ^{+} J(a r)\right)\right),
$$

where,

$$
J(r)=r \bar{N}\left(r, 1 / y_{0}^{(n-2)}\right)+r^{2} M_{1}(r) / A(r)
$$

Thus for special equations of the form (48), the growth of a meromorphic solution $y_{0}$ can be estimated in terms of the growth of the coefficients and the counting function for the zeros of $y_{0}^{(n-2)}$.

Proof. - Given $a>1$, let $\sigma>1$ be such that $\sigma^{n-1}=a$. Let $u_{0}=y_{0}^{(n-2)}$. Then $u_{0}$ satisfies equation (4), so there are constants $K, K_{1}$ and $r_{1}$ such that (5) and (6) hold for $r>r_{1}$ with $u_{0}$ replacing $y$ and $\sigma$ replacing $a$. By [5;p. 172], there are constants $K_{2}$ and $r_{0}>r_{1}$ such that for $r>r_{0}$, we have

$$
T\left(r, y_{0:}^{(j)}\right) \leqslant K_{2} T\left(\sigma r, y_{0}^{(j+1)}\right) \text { for } j=0,1, \ldots, n-3 .
$$

Thus $T\left(r, y_{0}\right) \leqslant K_{2}^{n-2} T\left(\sigma^{n-2} r, u_{0}\right)$ for $r>r_{0}$, which together with (5) and (6) (for $u_{0}$ in place of $y$ and $\sigma$ in place of $a$ ) yields the desired estimate (49) for $r>r_{0}$, where $C$ and $C_{1}$ are appropriate constants. 


\section{BIBLIOGRAPHY}

[1] S. BANK, A general theorem concerning the growth of solutions of first-order algebraic differential equations, Compositio Math., 25 (1972), pp. 61-70.

[2] S. BANK, On determining the growth of meromorphio solutions of algebraic differential equations having arbitrary entire coefficients, Nagoya Math. J., 49 (1973), pp. 53-65.

[3] S. BANK, A note on algebraic differential equations whose coefficients are entire functions of finite order, Ann. Scuola Norm. Sup. Pisa, 26 (1972), pp. $291-298$.

[4] O. Buumextrax, Principes de la théorie des fonetions entières d'ordre infini, GauthierVillars, Paris (1910).

[5] Chi-Tai Chuang, Sur la comparaison de la croissance d'une fonction méromorphe et de celle de sa derivée, Bull. Sci. Math., 75 (1951), pp. 171-190.

[6] MARGRIT FREI, Über die Lösungen linearer Differentialgleichungen mit ganzen Funktionem als Koeffizienten, Comment. Math. Helv., 35 (1961), pp. 201-222.

[7] W. K. Hayman, Meromorphic Funetions, Oxford Math. Monographs, Clarendon Press, Oxford (1964).

[8] S. Hellerstein - L. A. RuBes, Subfields that are algebraically closed in the field of all meromorphic funotions, J. Analyse Math., 12 (1964), pp. 105-111.

[9] R. NEvanlinna, Le théorème de Picard-Borel et la théorie des fonctions meromorphes, Gauthier-Villars, Paris (1929).

[10] S. SAKs - A. ZTGMUNd, Analytic Functions, Monografie Mat. (Engl. Transl.), tomo 28, Warsaw (1952).

[11] G. VALIRoN, Sur les fonctions entières verifiant wne olasse d'équations différentielles, Bull. Soc. Math. France, 51 (1923), pp. 33-45.

[12] G. Valinon, Lectures on the General Theory of Integral Functions, Edouard Privat, Toulouse (1923). 